\title{
Avaliação de um sistema simplificado de tratamento de esgotos visando a utilização em áreas rurais
}

\author{
Adriano L. Tonetti ${ }^{1}$, Bruno Coraucci Filho ${ }^{1}$, Edna I. Bertoncini ${ }^{1}$, Roberto A. Oliveira ${ }^{1}$ \& Ronaldo Stefanutti ${ }^{1}$
}

\section{RESUMO}

O Brasil enfrenta sérios problemas relacionados a uma grande população que vive na área rural, lançando os esgotos diretamente nos corpos hídricos. Este comportamento contribui a olhos vistos com a degradação do meio ambiente, além de trazer impasses à saúde pública; nesses casos, o emprego de sistemas de tratamento simplificados seria muito vantajoso, pois poderia depurar os esgotos dessas comunidades rurais a um custo baixo e ser operado e construído pela própria mão-de-obra local, embora pouco qualificada. Um dos sistemas estudados na UNICAMP e que se enquadra nessas características é, sem dúvida, a associação dos filtros anaeróbios com filtros de areia. Os resultados desta pesquisa demonstraram que o efluente gerado apresentava valores de matéria orgânica inferiores ao limite máximo estipulado pela legislação pertinente (CONAMA, 2005). Quanto aos compostos nitrogenados, demonstrou-se que os leitos de areia tinham grande capacidade de nitrificá-los e, no tocante ao fósforo, ressalta-se que nas maiores profundidades de leito de areia $(0,75$ e $1,00 \mathrm{~m})$, a remoção superava $98 \%$.

Palavras-chave: saneamento, nitrificação, fósforo, águas residuárias, purificação

\section{Evaluation of a simple system of wastewater treatment aiming at use in rural areas}

\begin{abstract}
Brazil faces problems related to the large population which lives in the rural areas and discharges the wastewater directly into water bodies. This occurrence contributes not only to environmental damage but also to public health problems. In this case, the use of simple treatments would be beneficial, since the local wastewater could be treated with low cost and be built and operated by local labor even though it is not specialized. A system investigated in UNICAMP and which has these characteristics is, undoubtedly, the association of anaerobic filters with sand filters. The results from the research showed that the effluent had an organic matter concentration lower than the maximum permitted by Brazilian law (Brasil, 2005). Regarding the nitrogen compounds, the sand filters showed a great capacity of nitrification and, in reference to phosphorus, in the deepest depths $(0.75$ and $1.00 \mathrm{~m})$, the removal was more than $98 \%$.
\end{abstract}

Key words: sanitation, nitrification, phosphorus, wastewater, purification

1 Faculdade de Engenharia Civil, Arquitetura e Urbanismo/UNICAMP, CP 6021, CEP 13083-970, Campinas, SP. Fone: 55 (19) 3521-2373. E-mail: altonetti@yahoo.com.br; bruno@fe.unicamp.br; ebertoncini@apta.sp.gov.br; ronaldo.stefanutti@pq.cnpq.br 


\section{INTRODUÇÃO}

Segundo o IBGE (2007), cerca de 18,71\% da população brasileira vivem na área rural, correspondendo a 31.845.211 habitantes que, em sua quase totalidade, lançam os esgotos diretamente nos rios, lagos ou mesmo no solo localizado nas proximidades das moradias. Este comportamento acaba piorando a contaminação dos corpos hídricos superficiais e subterrâneos, gerando sérios problemas à saúde pública e ao próprio ambiente em que vivem.

Segundo Silva \& Nour (2005), pouca atenção se dá ao tratamento de efluentes líquidos gerados nas propriedades rurais que, individualmente não produzem quantidade elevada de compostos poluidores, mas ao se considerar a sua totalidade apresentam um montante considerável, e são lançados de forma dispersa e sem o devido tratamento.

As técnicas simplificadas de tratamento de esgoto poderiam solucionar este problema, haja vista serem apropriadas às suas necessidades, exigindo pouca manutenção e baixo custo de instalação e operação; (Pell \& Nyberg, 1989; Prochaska \& Zouboulis, 2003), sua viabilidade pode ser constatada pela ampla utilização em países europeus, tais como a Suécia, Finlândia, Noruega e Dinamarca, nos quais aproximadamente 2,7 milhões de estabelecimentos a utilizam mas, segundo Kristiansen (1981) e Hamoda et al. (2004), a capacidade de purificação dessas formas de tratamento não é, até hoje, bem conhecida, levando a uma operação inadequada e a consequente degradação ambiental. Oliveira et al. (2001) afirmam que esta conduta pode agravar os problemas da eutrofização das águas superficiais e da contaminação dos aquíferos subterrâneos.

Para Tonetti et al. (2005), apesar da grande facilidade de aplicação dessas técnicas, deve-se fazer uma avaliação adequada dos impasses que seu uso pode ocasionar, buscando a melhoria dos aspectos construtivos e operacionais. Sempre é conveniente considerar que nos locais em que pode haver seu emprego, na maioria das situações existe uma população com baixo grau de instrução, com possibilidade de captar água para consumo nas proximidades do lançamento ou tratamento do próprio esgoto.

Ressalta-se que, no Brasil, cerca de 51\% do abastecimento público e privado são feitos por captação subterrânea através de mais de 200.000 poços tubulares e 1 milhão de cacimbas escavadas. No Estado de São Paulo 61\% dos núcleos urbanos se utilizam de aquíferos no fornecimento total ou parcial de água potável para uso público atingindo, em algumas bacias, cifras superiores a 90\%. Nas áreas rurais as águas subterrâneas também são amplamente exploradas constituindo-se no recurso mais econômico; esses poços são perfurados sem controle oferecendo, na maioria dos casos, um abastecimento não tratado e sem monitoração ou proteção sanitária (Foster, 1993).

A UNICAMP, apoiada pela rede temática PROSAB, tem um histórico de desenvolvimento de muitas pesquisas através das quais se busca contribuir para a resolução desta questão. Objetiva-se, com os métodos de tratamento estudados, tratar os esgotos de forma simplificada, barata e sustentada, sem a necessidade do envolvimento de pessoas especializa- das para sua construção e operação. Um dos sistemas pesquisados foi o tratamento de esgoto domésticos por filtros anaeróbios com recheio de bambu (Camargo \& Nour, 2001); Trata-se de um método que além de ser de baixo custo, consome pouca energia e produz uma quantidade pequena de lodo mas a remoção de organismos patogênicos, nutrientes e matéria orgânica, não atende aos padrões de lançamento da legislação brasileira. Procurando a complementação do tratamento, Tonetti et al. (2005) constataram que a associação deste reator a um filtro de areia seria uma alternativa que, sem dúvida, preservaria o baixo custo e ainda manteria mínima a necessidade de operação e manutenção.

Esse estudo doi realizado com o objetivo de verificar a eficácia da associação dos filtros de areia aos filtros anaeróbios com recheio de bambu, tendo-se em vista o tratamento de esgotos domésticos gerados em pequenas comunidades rurais. A avaliação deste sistema se deu por meio da análise de alguns parâmetros químicos e biológicos do efluente final, que foram comparados com os valores exigidos pela legislação ambiental brasileira (CONAMA, 2005). Para os parâmetros que a legislação nacional não determina valores específicos para o lançamento, buscou-se uma legislação estadual em que os mesmos estivessem inclusos; assim, para a DQO se empregou a legislação do Estado de Alagoas (Alagoas, 1985), para a concentração de fósforo adotou-se a do Estado do Rio Grande do Sul (CONSEMA, 2006) e, finalmente, para Escherichia coli se empregou a do Estado de São Paulo (CETESB, 1976).

\section{MATERIAL E MÉTODOS}

Este projeto foi instalado em uma área experimental situada na Estação de Tratamento de Efluente Graminha, na cidade de Limeira, Estado de São Paulo, sendo que o esgoto bruto empregado na pesquisa era proveniente de um bairro residencial cuja população era de aproximadamente 8.000 pessoas.

\section{Filtros anaeróbios}

Uma parcela do esgoto bruto que chegava à Estação de Tratamento tinha seu fluxo direcionado para quatro filtros anaeróbios, de formato cilíndrico e volume de $500 \mathrm{~L}$, com diâmetro de 0,75 m e altura de 1,68 m, onde era parcialmente tratado. O meio suporte deste reator se compunha de anéis de bambu da espécie Bambusa tuldoides, com diâmetro de $0,04 \mathrm{~m}$ e comprimento de $0,05 \mathrm{~m}$. A operação se dava com fluxo ascendente e tempo de detenção hidráulica de 6 h (Camargo \& Nour, 2001). Na Figura 1A se encontra o esquema de um desses filtros anaeróbios e, nas Figuras 1B e 1C, respectivamente, as vistas interna e externa.

\section{Filtros de areia}

Após passar pelos filtros anaeróbios, denominados FB, parte do efluente era encaminhada para uma caixa de dosagem na qual se media o volume correspondente a cada carga de aplicação, para posterior disposição sobre os quatro filtros de areia. Esses filtros de areia, por sua vez, foram 
A.

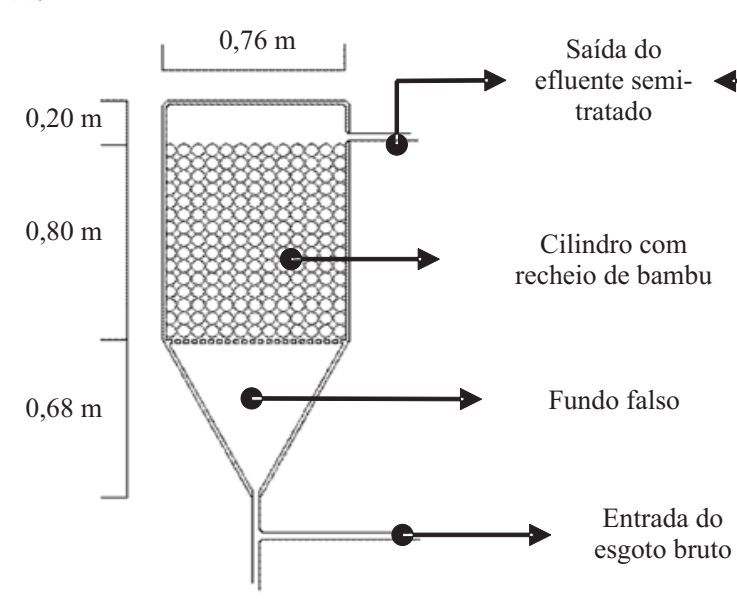

B.

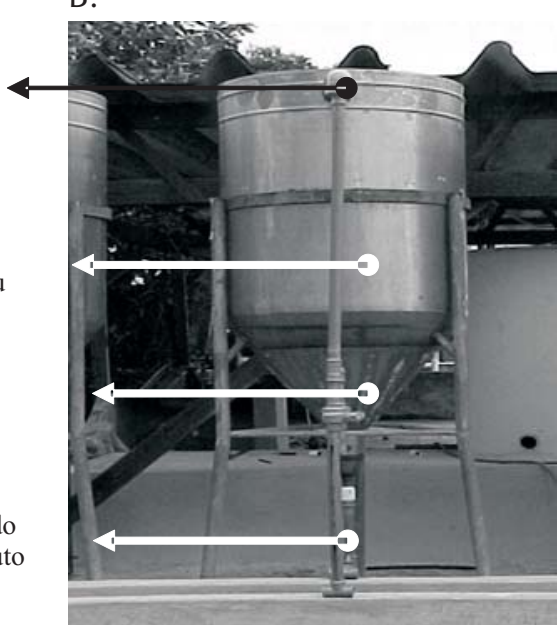

C.

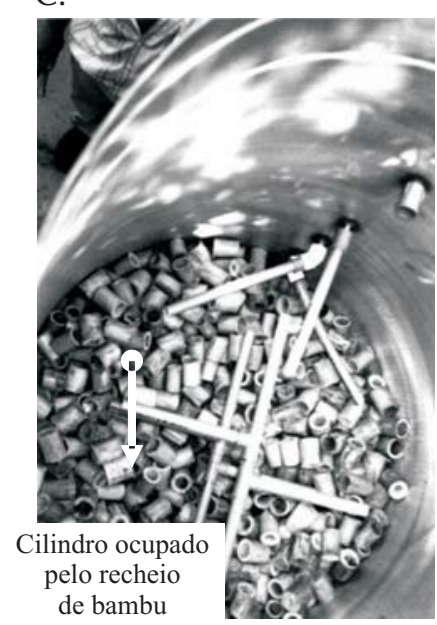

Figura 1. Esquema de um filtro anaeróbio com recheio de bambu (A) e vista externa (B) e interna (C)

construídos em caixas cilíndricas de fibra de vidro, com diâmetro interno de 1,00 m e no interior de cada uma se dispuseram três camadas a partir da base, conforme a Figura 2 .

A primeira camada, de $0,20 \mathrm{~m}$ de profundidade, foi constituída de brita 4 de diâmetro efetivo $\left(\mathrm{D}_{10}\right)$ de $37,000 \mathrm{~mm}$ e coeficiente de desuniformidade $\left(\mathrm{C}_{\mathrm{D}}\right)$ de 2,400; logo acima estava a camada de brita 1 , com $D_{10}$ igual a $9,600 \mathrm{~mm}, C_{D}$ de 1,771 e $0,10 \mathrm{~m}$ de profundidade, materiais que objetivavam sustentar a areia, impedindo seu arraste para fora do sistema. Para a areia, que possuía $\mathrm{D}_{10}$ de $0,093 \mathrm{~mm}$ e $\mathrm{C}_{\mathrm{D}}$ de 4,516, em cada caixa se adotou uma espessura diferente, conforme apresentado na Tabela 1.

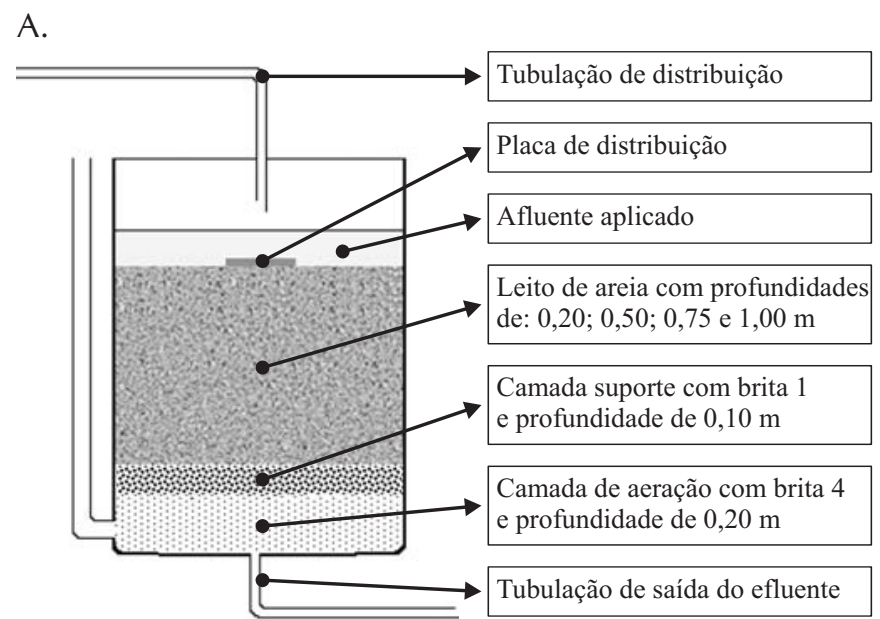

B.

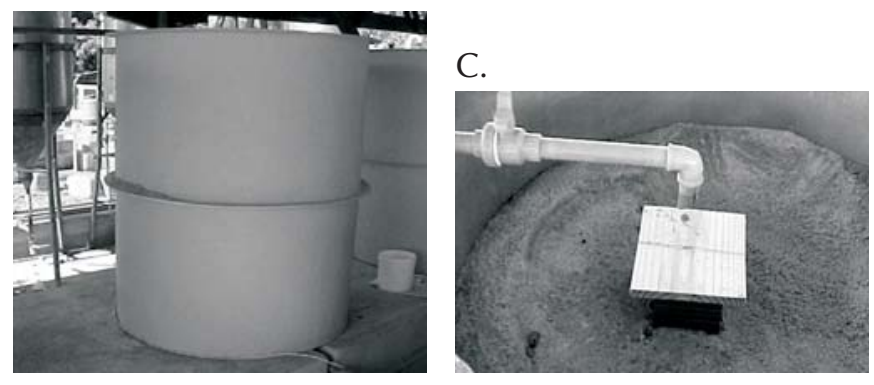

Figura 2. Esquema dos filtros de areia (A), vista geral (B) e vista da superfície (C)
Tabela 1. Denominação e profundidade dos filtros de areia

\begin{tabular}{cc}
\hline Filtro & Profundidade do filtro de areia $(\mathbf{m})$ \\
F025 & 0,25 \\
F050 & 0,50 \\
F075 & 0,75 \\
F100 & 1,00 \\
\hline
\end{tabular}

Buscando-se ampliar a facilidade construtiva desta tecnologia, a areia empregada no preenchimento desses filtros foi a popularmente denominada grossa, cuja distribuição granulométrica está apresentada na Tabela 2.

Tabela 2. Distribuição granulométrica da areia empregada nos filtros

\begin{tabular}{|c|c|c|c|c|c|c|c|}
\hline $\begin{array}{c}\text { Argila } \\
(\%)\end{array}$ & $\begin{array}{l}\text { Silte } \\
(\%)\end{array}$ & & $\begin{array}{c}\text { Areia } \\
(\%)\end{array}$ & & & $\begin{array}{l}\text { edregulh } \\
\text { (\%) }\end{array}$ & \\
\hline \multirow[b]{2}{*}{0,0} & \multirow[b]{2}{*}{0,0} & \multicolumn{3}{|c|}{90,0} & \multicolumn{3}{|c|}{10} \\
\hline & & $\begin{array}{l}\text { Fina } \\
26.9\end{array}$ & $\begin{array}{c}\text { Média } \\
42.3\end{array}$ & $\begin{array}{c}\text { Grossa } \\
20.7\end{array}$ & $\begin{array}{c}\text { Fino } \\
4.9\end{array}$ & $\begin{array}{c}\text { Médio } \\
5.1\end{array}$ & $\begin{array}{c}\text { Grosso } \\
0.0\end{array}$ \\
\hline
\end{tabular}

A área em que se encontrava a instalação deste sistema de tratamento, era toda coberta, impedindo a interferência da precipitação pluviométrica. Neste local, a temperatura média durante os dois anos de desenvolvimento do projeto era de $21,1^{\circ} \mathrm{C}$; destaca-se também que o experimento foi montado em esquema fatorial 4 x 5 x 3 (profundidade do leito $\mathrm{x}$ carga aplicada $\mathrm{x}$ frequência/dia) e conduzido pelo tempo de dezoito meses.

\section{Cargas de efluente anaeróbio aplicado nas superfícies dos filtros}

Nas superfícies dos filtros de areia aplicou-se o efluente dos filtros anaeróbios em curto intervalo de tempo, nas cargas de 20, 40, 60, 80 e $100 \mathrm{~L} \mathrm{~m}^{-2}$; todas essas cargas foram utilizadas em três etapas diferentes: na primeira etapa, cada uma delas foi aplicada uma vez por dia, às $8 \mathrm{~h}$ da manhã, em cada um dos filtros de areia; durante a segunda etapa ocorreram duas disposições relacionadas a essas cargas, em dois horários intervalados por uma hora, ou seja, 
às 8 e às 9 h; finalmente, na terceira etapa se aplicaram, diariamente e em três momentos, as cargas, observando-se o mesmo espaço de uma hora entre elas. Destaca-se que, em suas respectivas frequências, cada carga foi empregada durante um mês.

Em uma situação modelo a carga de $20 \mathrm{~L} \mathrm{~m}^{-2}$ foi aplicada uma única vez por dia, na primeira etapa, durante um mês, ao final do qual se iniciou o emprego de $40 \mathrm{~L} \mathrm{~m}^{-2}$, pelo mesmo prazo de estudo cujo procedimento se repetiu até a disposição de $100 \mathrm{~L} \mathrm{~m}^{-2}$; logo após se iniciou a segunda etapa com a aplicação das mesmas cargas duas vezes ao dia perfazendo, no caso da carga de $20 \mathrm{~L} \mathrm{~m}^{-2}$, uma taxa de $40 \mathrm{~L} \mathrm{~m}^{-2} \mathrm{~d}^{-1}$; por fim, na última etapa ocorreram três aplicações diárias totalizando, no exemplo, uma taxa de $60 \mathrm{~L} \mathrm{~m}^{-2} \mathrm{~d}^{-1}$. Na Tabela 3 se apresentam para cada etapa, a carga empregada, sua frequência e respectiva taxa diária.

Tabela 3. Cargas aplicadas nas diferentes etapas e correspondência com a taxa diária

\begin{tabular}{|c|c|c|c|c|c|}
\hline \multicolumn{2}{|c|}{$\begin{array}{c}\text { Etapa } 1 \\
1 \text { Aplicação por dia }\end{array}$} & \multicolumn{2}{|c|}{$\begin{array}{c}\text { Etapa } 2 \\
2 \text { Aplicações por dia }\end{array}$} & \multicolumn{2}{|c|}{$\begin{array}{c}\text { Etapa } 3 \\
3 \text { Aplicações por dia }\end{array}$} \\
\hline $\begin{array}{l}\text { Carga } \\
\left(\mathrm{L} \mathrm{m}^{-2}\right)\end{array}$ & $\begin{array}{c}\text { Taxa diária } \\
\text { equivalente } \\
\left(\mathrm{L} \mathrm{m}^{-2} \mathrm{~d}^{-1}\right)\end{array}$ & $\begin{array}{l}\text { Carga } \\
\left(\mathrm{L} \mathrm{m}^{-2}\right)\end{array}$ & $\begin{array}{c}\text { Taxa diária } \\
\text { equivalente } \\
\left(\mathrm{L} \mathrm{m}^{-2} \mathrm{~d}^{-1}\right)\end{array}$ & $\begin{array}{l}\text { Carga } \\
\left(\mathrm{L} \mathrm{m}^{-2}\right)\end{array}$ & $\begin{array}{l}\text { Taxa diária } \\
\text { equivalente } \\
\text { ( }\left(\mathrm{m}^{-2} \mathbf{d}^{-1}\right)\end{array}$ \\
\hline 20 & 20 & 20 & 40 & 20 & 60 \\
\hline 40 & 40 & 40 & 80 & 40 & 120 \\
\hline 60 & 60 & 60 & 120 & 60 & 180 \\
\hline 80 & 80 & 80 & 160 & 80 & 240 \\
\hline 100 & 100 & 100 & 200 & 100 & 300 \\
\hline
\end{tabular}

\section{Coleta de amostras e análises laboratoriais}

O efluente dos filtros anaeróbios e dos filtros de areia foi coletado semanalmente, logo após a aplicação da última carga diária, sendo que as análises da demanda química de oxigênio (DQO), fósforo e E. Coli, se basearam no Standard Methods for the Examination of Water and Wastewater (APHA/ AWWA/WEF, 1998). Fez-se a determinação de E. Coli imediatamente após a coleta das amostras, por meio do teste do substrato cromogênico definido, ocorrendo a confirmação para a presença desses microrganismos em 24 h, pelo desenvolvimento de fluorescência.

Empregou-se, na determinação de fósforo, uma vidraria previamente lavada com $\mathrm{HCl}$ 1:1 sendo que todas as amostras foram digeridas com $\mathrm{H}_{2} \mathrm{SO}_{4}$ e $\mathrm{HNO}_{3}$ concentrados a uma temperatura de $105^{\circ} \mathrm{C}$, pelo tempo de $1 \mathrm{~h}$; logo após, resfriou-se o líquido e se neutralizou com $\mathrm{NaOH} 6 \mathrm{~mol} \mathrm{~L}^{-1}$ determinando-se, no final, a concentração colorimetricamente depois da formação do complexo fosfomolibdico reduzido, no comprimento de onda de $880 \mathrm{~nm}$.

Para se encontrar os valores de nitrogênio total Kjeldahl (N-NTK), que é a soma do nitrogênio amoniacal e orgânico, efetuou-se a digestão com uma mistura de $2 \mathrm{~mL}$ de $\mathrm{H}_{2} \mathrm{SO}_{4}$ concentrado, sulfato de potássio e sulfato de mercúrio, com aquecimento a $350{ }^{\circ} \mathrm{C}$. Por fim, o extrato obtido era encaminhado para a análise, por injeção de fluxo (Faria \& Pasquini, 1991).

$\mathrm{O}$ procedimento para a determinação de N-nitrato e $\mathrm{N}$ nitrito foi baseado na redução desses compostos para $\mathrm{NH}_{4}^{+}$ através de uma coluna de $\mathrm{Zn}^{0}$, após a alcalinização da amostra com solução de $\mathrm{NaOH} 0,1 \mathrm{~mol} \mathrm{~L}^{-1}$. As formas de nitrogênio amoniacal se difundem através de uma membrana de polipropileno e atingem uma cela de condutividade, possibilitando inferir sua relação com a concentração.

Os dados de DQO (demanda química de oxigênio), fósforo e E. coli, foram submetidos a análise de variância e teste de comparação de médias por Tukey 5\%, através do programa estatístico SANEST (Zonta, 1984).

\section{RESULTADOS E DISCUSSÃO}

\section{Demanda química de oxigênio - DQO}

Neste item se comparam os resultados das concentrações de DQO dos efluentes dos filtros de areia com a legislação do Estado de Alagoas (Alagoas, 1985), que impõe o limite máximo de $150 \mathrm{mg} \mathrm{L}^{-1}$, visto que não existe uma normatização nacional quanto a este parâmetro.

Averigua-se, na Tabela 4, que na média o F025 apresentou um conjunto de seis resultados, que ultrapassaram o limite da legislação alagoana, demonstrando a grande dificuldade da profundidade de leito adotada neste filtro em propiciar concentrações adequadas de DQO, principalmente no emprego das maiores frequências de aplicações.

Quanto aos F075 e F100, em nenhum caso foram geraram resultados acima da legislação de Alagoas, permitindo comprovar sua grande eficiência na remoção de matéria orgânica, o que pode ser explicado pela profundidade de leito maior, que permitiu a ampliação da filtração e a adesão de uma grande quantidade de bactérias responsáveis pela depuração biológica, fato que demonstra, tal como o afirmado por Kristiansen (1981), que este sistema deve ser considerado um filtro vivo. Para o F050, os resultados também estiveram abaixo do valor estipulado, exceto para a carga de $100 \mathrm{~L} \mathrm{~m}^{-2}$ com frequência de duas e três vezes ao dia.

Ao se analisar os dados da etapa 1 percebe-se que, conforme se aumentaram os valores das cargas aplicadas, passou a existir uma diferenciação no tratamento propiciado pelos filtros de areia. Na aplicação de $20 \mathrm{~L} \mathrm{~m}^{-2}$ todas as profundidades de leito geraram efluentes cujas concentrações médias não tinham diferenças significativas indicando que na aplicação de baixos valores de afluente a escolha de uma profundidade específica de leito de areia não é fator determinante.

Nota-se, na aplicação de $100 \mathrm{~L} \mathrm{~m}^{-2}$, que: o F025 tinha resultados significativamente diferentes dos outros três filtros; os F050 e F075 apresentaram concentrações que não mostravam diferenças significativas entre si e, no F100, a DQO era a mais baixa e significativamente diferente dos outros três casos estudados, comportamento este que demonstra que, quando se aumentou a carga aplicada, a profundidade de leito de areia empregada passou a influenciar no tratamento. já na etapa 2 se ampliou esta característica fazendo com que, na aplicação de $100 \mathrm{~L} \mathrm{~m}^{-2}$, os quatro filtros de areia passassem a ter efluentes concentrações significativamente diferentes.

Ao se analisar os percentuais de remoção, constata-se a 
Tabela 4. Concentração de DQO (mg L $\left.\mathrm{L}^{-1}\right)$ nos efluentes dos filtros de areia e filtros anaeróbios ${ }^{(1)}$

\begin{tabular}{|c|c|c|c|c|c|c|c|c|c|}
\hline Carga $\left(\mathrm{Lm}^{-2}\right)$ & $\mathrm{FB}^{3}$ & F025 & $\mathbf{R}^{2}$ & F050 & $\mathbf{R}$ & F075 & $\mathbf{R}$ & F100 & $\mathbf{R}$ \\
\hline \multicolumn{10}{|c|}{ Etapa 1 - 1 Aplicação por dia } \\
\hline 20 & 415 & $20 \mathrm{a}$ & 95,0 & $28 \mathrm{a}$ & 93,3 & $36 \mathrm{a}$ & 91,3 & $18 \mathrm{a}$ & 95,6 \\
\hline 40 & 363 & $86 \mathrm{a}$ & 76,2 & $91 \mathrm{~b}$ & 74,8 & $29 \mathrm{c}$ & 91,8 & $25 \mathrm{c}$ & 93,0 \\
\hline 60 & 526 & 159 a & 69,8 & $64 \mathrm{~b}$ & 87,8 & $91 \mathrm{~b}$ & 82,7 & $74 \mathrm{~b}$ & 85,9 \\
\hline 80 & 439 & $109 \mathrm{a}$ & 75,2 & $89 \mathrm{~b}$ & 79,7 & $65 \mathrm{~b}$ & 85,1 & $67 c$ & 84,6 \\
\hline 100 & 343 & $103 \mathrm{a}$ & 69,8 & $58 \mathrm{~b}$ & 83,0 & $35 \mathrm{~b}$ & 89,5 & $21 \mathrm{c}$ & 93,9 \\
\hline \multicolumn{10}{|c|}{ Etapa 2 - 2 Aplicações por dia } \\
\hline 20 & 351 & $25 \mathrm{a}$ & 92,8 & $21 a b$ & 94,0 & $16 \mathrm{~b}$ & 95,3 & $17 \mathrm{~b}$ & 94,9 \\
\hline 40 & 356 & $51 \mathrm{a}$ & 85,5 & $26 \mathrm{~b}$ & 92,5 & $21 \mathrm{c}$ & 94,1 & $28 \mathrm{bc}$ & 92,2 \\
\hline 60 & 565 & $93 \mathrm{a}$ & 83,4 & $95 \mathrm{~b}$ & 83,2 & $40 \mathrm{c}$ & 92,8 & $56 d$ & 90,0 \\
\hline 80 & 604 & $144 \mathrm{a}$ & 76,1 & $115 b$ & 81,0 & $84 \mathrm{c}$ & 86,1 & $73 d$ & 87,9 \\
\hline 100 & 551 & $200 \mathrm{a}$ & 63,7 & $150 \mathrm{~b}$ & 72,7 & $101 \mathrm{c}$ & 81,5 & $80 \mathrm{~d}$ & 85,3 \\
\hline \multicolumn{10}{|c|}{ Etapa 3 - 3 Aplicações por dia } \\
\hline 20 & 558 & 77 a & 86,1 & $38 \mathrm{~b}$ & 93,2 & $24 \mathrm{c}$ & 95,7 & $7 \mathrm{c}$ & 98,6 \\
\hline 40 & 413 & 94 a & 77,2 & $42 \mathrm{~b}$ & 89,8 & $32 \mathrm{bc}$ & 92,2 & $12 \mathrm{c}$ & 97,0 \\
\hline 60 & 382 & $176 \mathrm{a}$ & 53,8 & $130 \mathrm{~b}$ & 65,8 & $89 \mathrm{c}$ & 76,6 & $64 d$ & 83,0 \\
\hline 80 & 434 & $169 \mathrm{a}$ & 60,9 & $142 \mathrm{~b}$ & 67,2 & $119 \mathrm{c}$ & 72,6 & $120 \mathrm{~d}$ & 72,3 \\
\hline 100 & 484 & $164 \mathrm{a}$ & 66,1 & $155 \mathrm{a}$ & 67,9 & $119 \mathrm{bc}$ & 75,4 & $93 \mathrm{c}$ & 80,8 \\
\hline
\end{tabular}

${ }^{1}$ Médias seguidas da mesma letra minúscula na horizontal, não diferem entre si (Tukey 5\%); ${ }^{2}$ Remoção em relação à concentração do efluente dos filtros anaeróbios; ${ }^{3}$ Apesar de não haver mudanças na operação dos filtros anaeróbios (FB), fez-se a média para as concentrações de DQO no período compreendido por cada carga de aplicação nos filtros de areia

grande eficiência deste sistema de tratamento. O F100 teve durante a primeira etapa uma remoção mínima de 84,6\% e, na terceira etapa, chegou a superar 98,0\%. Destaca-se que os valores apresentados na Tabela 4 se referem à diminuição da concentração de DQO em relação aos valores encontrados no efluente dos filtros anaeróbios (FB) e, caso fossem somadas as remoções propiciadas pelos filtros de areia e pelos filtros anaeróbios, ver-se-iam resultados ainda melhores.

\section{Compostos nitrogenados}

Devido a limitação de espaço, neste item somente serão apresentadas e discutidas as concentrações de nitrogênio total Kjeldahl (N-NTK), que é a soma do nitrogênio amonia- cal e orgânico, e o nitrogênio total, correspondente ao somatório do N-NTK com os valores de N-nitrato e N-nitrito.

Inicialmente, se encontrou que no efluente dos filtros anaeróbios, em todos os casos estudados a soma da concentração de $\mathrm{N}$-nitrato e $\mathrm{N}$-nitrito jamais atingiu $1 \mathrm{mg} \mathrm{L}^{-1}$; deste modo, quanto aos compostos nitrogenados havia apenas a presença quase que exclusiva de nitrogênio total Kjeldahl.

Nota-se, na Tabela 5, que na primeira etapa durante a aplicação de $20 \mathrm{~L} \mathrm{~m}^{-2}$, a concentração do nitrogênio total presente no efluente dos filtros anaeróbios era muito superior àquelas presentes nos efluentes dos filtros de areia cujo comportamento pode ser resultante da adsorção dos compostos nitrogenados pelos leitos de areia que, no decorrer do

Tabela 5. Média da concentração dos compostos nitrogenados (mg L-1) nos efluentes dos filtros F025, F050, F075 e F100 e no filtro anaeróbio

\begin{tabular}{|c|c|c|c|c|c|c|c|c|c|}
\hline \multirow{2}{*}{ Carga $\left(\mathrm{L} \mathrm{m}^{-2}\right)$} & \multirow{2}{*}{$\begin{array}{c}\text { Filtros } \\
\text { Anaeróbios } \\
\text { N-NTK }\end{array}$} & \multicolumn{2}{|c|}{ F025 } & \multicolumn{2}{|c|}{ F050 } & \multicolumn{2}{|c|}{ F075 } & \multicolumn{2}{|c|}{ F100 } \\
\hline & & N-NTK ${ }^{1}$ & $\mathrm{~N}-\mathrm{N}_{\mathrm{T}}^{2}$ & N-NTK & $\mathrm{N}-\mathrm{N}_{\mathrm{T}}$ & N-NTK & $\mathrm{N}-\mathrm{N}_{\mathrm{T}}$ & N-NTK & $\mathrm{N}-\mathrm{N}_{\mathrm{T}}$ \\
\hline \multicolumn{10}{|c|}{ Etapa 1 - 1 Aplicação por dia } \\
\hline 20 & 44,0 & n.d. ${ }^{3}$ & 10,1 & n.d. & 11,3 & n.d. & 7,6 & n.d. & 13,9 \\
\hline 40 & 69,7 & 1,7 & 56,1 & n.d. & 55,6 & n.d. & 54,0 & n.d. & 51,2 \\
\hline 60 & 92,4 & 8,3 & 65,3 & 1,2 & 56,9 & 3,1 & 65,7 & n.d. & 54,6 \\
\hline 80 & 86,2 & 13,5 & 80,5 & 6,6 & 79,9 & 3,9 & 78,8 & 1,9 & 91,3 \\
\hline 100 & 79,6 & 6,4 & 98,9 & 3,1 & 83,0 & 1,7 & 55,8 & 1,2 & 63,7 \\
\hline \multicolumn{10}{|c|}{ Etapa 2 - 2 Aplicações por dia } \\
\hline 20 & 56,2 & n.d. & 57,9 & n.d. & 54,5 & n.d & 58,8 & n.d. & 64,7 \\
\hline 40 & 40,1 & 2,9 & 30,1 & 1,6 & 34,9 & 1,0 & 35,4 & n.d. & 39,0 \\
\hline 60 & 67,7 & 7,3 & 61,9 & 2,9 & 41,1 & 3,9 & 49,4 & 1,9 & 46,3 \\
\hline 80 & 109,7 & 11,3 & 24,6 & 9,6 & 40,7 & 8,6 & 50,3 & 13,2 & 46,4 \\
\hline 100 & 84,3 & 60,6 & 75,3 & 65,0 & 105,3 & 56,9 & 99,6 & 39,4 & 80,2 \\
\hline \multicolumn{10}{|c|}{ Etapa 3 - 3 Aplicações por dia } \\
\hline 20 & 87,8 & 6,2 & 65,6 & 2,5 & 73,6 & 2,8 & 76,9 & 3,0 & 78,8 \\
\hline 40 & 130,5 & 9,7 & 42,8 & 5,3 & 59,7 & 4,0 & 60,6 & 2,6 & 78,4 \\
\hline 60 & 104,3 & 19,5 & 29,6 & 9,4 & 26,5 & 8,8 & 60,1 & 5,9 & 62,7 \\
\hline 80 & 113,1 & 65,1 & 68,7 & 27 & 45,5 & 24,5 & 58,6 & 24,0 & 52,1 \\
\hline 100 & 68,7 & 49,6 & 52,7 & 42,2 & 47,2 & 27,3 & 41,0 & 19,9 & 46,6 \\
\hline
\end{tabular}

${ }^{1}$ Nitrogênio Total Kjeldahl, soma do nitrogênio amoniacal e orgânico; ${ }^{2}$ Soma do nitrogênio orgânico com 0 amoniacal, nitrato e nitrito; ${ }^{3}$ Não detectado pela técnica empregada na análise 
desenvolvimento da pesquisa, foi perdendo o desempenho nesta função. Na disposição de 80 e $100 \mathrm{~L} \mathrm{~m}^{-2}$, durante esta mesma etapa, passou a haver tendência ao igualamento da concentração do nitrogênio total no afluente e no efluente dos filtros; tal comportamento está de acordo com o exposto por Check et al. (1994) que propuseram que os compostos nitrogenados acabam sendo adsorvidos pelo leito de areia durante a percolação do afluente.

Nesta primeira etapa ocorreu, nos filtros F025 e F050, um forte processo de nitrificação que foi ainda mais ampliado no F075 e F100. Nesses filtros com camada de leito mais profunda se propiciava um contato maior do substrato com os microrganismos aderidos no grão de areia, possibilitando uma transformação dos compostos nitrogenados ainda mais acentuada; ainda neles, a média de N-NTK nunca superou 3,9 $\mathrm{mg} \mathrm{L}^{-1}$, mesmo no emprego de 80 e $100 \mathrm{~L} \mathrm{~m}^{-2}$. Deste modo, em todos os casos seria atendida a legislação brasileira (Brasil, 2005) quanto ao lançamento em corpos hídricos, a qual estabelece o limite de $20,0 \mathrm{mg} \mathrm{L}^{-1}$ para a concentração de N-NTK, em termos de nitrogênio amoniacal total

Durante a segunda etapa também se deu, nos filtros F025 e F050 na aplicação de 20, 40 e $60 \mathrm{~L} \mathrm{~m}^{-2}$, uma grande nitrificação mas, a partir da disposição de $100 \mathrm{~L} \mathrm{~m}^{-2}$, a média de N-NTK constituía mais da metade do nitrogênio total presente no lixiviado, característica que indica um possível limite do tratamento desses filtros. Para os filtros F075 e F100, somente na carga de $100 \mathrm{~L} \mathrm{~m}^{-2}$ a concentração de nitrogênio total Kjeldahl atingiu 56,9 $\mathrm{mg} \mathrm{L}^{-1}$, superando a metade da concentração de nitrogênio total; para os F025 e F050, na terceira etapa, este aumento da concentração de nitrogênio total Kjeldahl se iniciou na carga de $60 \mathrm{~L} \mathrm{~m}^{-2}$, sendo que na disposição de 80 e $100 \mathrm{~L} \mathrm{~m}^{-2}$ correspondia a 94,0\% de todo o nitrogênio total que era lixiviado, ou seja, nessas condições não havia, praticamente, tratamento durante a infiltração do efluente anaeróbio, mas sim quanto à legislação brasileira. No caso dos filtros F075 e F100 na aplicação de
80 e $100 \mathrm{~L} \mathrm{~m}^{-2}$, apesar da nitrificação ter sido reduzida ainda estava presente, embora o N-NTK nunca tenha atingido valores que superassem a metade da concentração do nitrogênio total.

De forma geral e após o período inicial de operação do sistema, constata-se que o nitrogênio total que era aplicado independentemente da profundidade do filtro, acabava sofrendo uma mineralização, mesmo que parcial, e deixava o sistema com concentrações bastante próximas à do afluente, indicando o não acúmulo no filtro. A grande distinção entre as diferentes profundidades de leito estudadas se refere à capacidade de nitrificação do afluente disposto. Ressalta-se que os filtros com leitos mais profundos tiveram excelente desempenho em toda a primeira etapa e, na segunda, sua grande eficiência chegou até à aplicação de $80 \mathrm{~L} \mathrm{~m}^{-2}$.

Para Sousa et al. (2006), a remoção de nutrientes é fundamental para atenuar o processo de eutrofização quando as águas superficiais são o destino final do efluente porém, quando o mesmo se destina à irrigação, o interesse é manter altas concentrações de nitrogênio, uma vez que este elemento substituirá os fertilizantes químicos; assim se teria que o efluente gerado pelos filtros de areia apresentaria grande potencial para então ser reutilizado na agricultura, devido principalmente à sua alta concentração de nitrato.

\section{Fósforo}

Na primeira etapa a média das concentrações de fósforo no efluente dos filtros anaeróbios foi de 11,8 $\mathrm{mg} \mathrm{L}^{-1}$; após a aplicação nos filtros de areia, como pode ser visualizado na Tabela 6, ocorreu uma grande redução de seus valores, podendo-se dividir, estatisticamente, os dados em dois grupos com resultados significativamente diferentes, em que em um deles estaria o F025 que, excetuando-se os valores obtidos na disposição de $20 \mathrm{~L} \mathrm{~m}^{-2}$ teve, isoladamente, em todos os outros casos, isoladamente, as maiores concentrações de fósforo; no outro grupo estariam os F050, F075 e F100, com

Tabela 6. Concentração média de fósforo $\left(\mathrm{mg}^{-1}\right)$ no efluente dos filtros de areia e filtro anaeróbio $(\mathrm{FB})^{(1)}(2)$

\begin{tabular}{|c|c|c|c|c|c|c|c|c|c|}
\hline Carga $\left(\mathrm{L} \mathrm{m}^{-2}\right)$ & FB & F025 & $R^{4}$ & F050 & $\mathbf{R}$ & F075 & $\mathbf{R}$ & F100 & $\mathbf{R}$ \\
\hline \multicolumn{10}{|c|}{ Etapa 1 - 1 Aplicação por dia } \\
\hline 20 & 10,6 & $0,3 \mathrm{aA}$ & 96,6 & $0,2 \mathrm{aA}$ & 97,6 & $0,1 \mathrm{aA}$ & 98,3 & $0,2 \mathrm{aA}$ & 97,3 \\
\hline 40 & 9,1 & 0,5 aAB & 95,3 & $0,1 \mathrm{bA}$ & 99,1 & $0,1 \mathrm{bA}$ & 99,1 & $0,1 \mathrm{bA}$ & 99,5 \\
\hline 60 & 19,8 & 4,3 aCD & 70,0 & $0,3 \mathrm{bA}$ & 98,2 & $0,1 \mathrm{bA}$ & 99,5 & $0,1 \mathrm{bA}$ & 99,6 \\
\hline 80 & 17,1 & $3,6 \mathrm{aD}$ & 72,1 & $1,0 \mathrm{bA}$ & 92,6 & $0,5 \mathrm{cA}$ & 96,3 & $0,2 \mathrm{cA}$ & 98,7 \\
\hline 100 & 14,7 & $1,9 \mathrm{aBC}$ & 83,3 & $0,6 \mathrm{bA}$ & 95,0 & $0,4 \mathrm{bA}$ & 96,2 & $0,1 \mathrm{bA}$ & 98,7 \\
\hline 20 & 11,8 & $0,3 \mathrm{aA}$ & 98,0 & $0,1 \mathrm{aA}$ & 99,2 & $0,1 \mathrm{aA}$ & 99,2 & $0,1 \mathrm{aA}$ & 99,6 \\
\hline 40 & 18,4 & $1,4 \mathrm{aAB}$ & 81,0 & $0,3 \mathrm{bA}$ & 95,5 & $0,7 \mathrm{aA}$ & 90,1 & $0,1 \mathrm{bA}$ & 98,8 \\
\hline 60 & 10,6 & $1,8 \mathrm{aB}$ & 81,5 & $0,9 \mathrm{bA}$ & 90,9 & $0,8 \mathrm{bA}$ & 91,5 & n.d. cA & 99,8 \\
\hline 80 & 13,0 & $4,3 \mathrm{aC}$ & 54,2 & $4,2 \mathrm{bB}$ & 55,1 & $3,0 \mathrm{aB}$ & 67,6 & $1,5 \mathrm{cAB}$ & 84,2 \\
\hline 100 & 12,0 & $7,6 \mathrm{aD}$ & 25,7 & $3,7 \mathrm{bB}$ & 64,3 & $3,7 \mathrm{cB}$ & 64,3 & $2,2 \mathrm{~dB}$ & 78,1 \\
\hline \multicolumn{10}{|c|}{ Etapa 3 - 3 Aplicações por dia } \\
\hline 20 & 26,4 & $3,1 \mathrm{aA}$ & 86,5 & $1,5 \mathrm{bA}$ & 93,6 & $1,0 \mathrm{bA}$ & 95,5 & $0,3 \mathrm{cA}$ & 98,8 \\
\hline 60 & 14,0 & $8,0 \mathrm{aC}$ & 8,1 & $4,4 \mathrm{bB}$ & 48,7 & $3,6 \mathrm{cB}$ & 58,9 & $3,0 \mathrm{~dB}$ & 65,2 \\
\hline 80 & 15,4 & $5,8 \mathrm{aB}$ & 35,1 & $7,3 \mathrm{bB}$ & 18,2 & $5,9 \mathrm{cB}$ & 34,5 & $4,8 \mathrm{cB}$ & 46,1 \\
\hline 100 & 15,5 & $10,8 \mathrm{aD}$ & 22,1 & $8,4 \mathrm{bC}$ & 39,4 & 9,8 bc & 29,7 & $6,5 \mathrm{cC}$ & 53,7 \\
\hline
\end{tabular}

${ }^{1}$ Médias seguidas da mesma letra minúscula na horizontal não diferem entre si (Tukey $5 \%$ ); ${ }^{2}$ Médias seguidas da mesma letra maiúscula na vertical não diferem entre si em uma mesma etapa (Tukey $5 \%) ;{ }^{3}$ Remoção em relação à concentração do efluente dos filtros anaeróbios 
um lixiviado sem diferenças significativas para as médias de concentração de fósforo.

Nesta primeira etapa durante a disposição de $100 \mathrm{~L} \mathrm{~m}^{-2}$, todos os filtros apresentaram diminuição da concentração de fósforo lixiviado quando comparado com a aplicação de $80 \mathrm{~L} \mathrm{~m}^{-2}$; a provável explicação para tal comportamento, pode ser a formação de uma maior estrutura de biofilme, a qual ampliou a capacidade de adsorver o fósforo presente no afluente.

Durante as segunda e terceira etapas, o F025 apresentava o mesmo comportamento da primeira. O F100 consolidouse como o filtro que tinha a maior capacidade de reter o fósforo, principalmente durante o emprego de 60, 80 e $100 \mathrm{~L} \mathrm{~m}^{-2}$, quando seus valores médios foram significativamente diferentes daqueles obtidos para os outros três filtros.

Analisando-se o comportamento de cada filtro com o aumento das cargas aplicadas, percebe-se que os F050, F075 e F100 tiveram concentrações que não foram estatisticamente diferentes até o emprego de $60 \mathrm{~L} \mathrm{~m}^{-2}$, na segunda etapa; assim, esses filtros tiveram maior capacidade em suportar o aumento do volume de afluente sem acarretar em acréscimo na concentração de fósforo no líquido lixiviado; já na terceira etapa deu-se uma mudança neste comportamento e os F050, F075 e F100 passaram a apresentar três níveis significativamente diferentes para as concentrações médias de fósforo, em que no primeiro estariam os resultados das cargas de 20 e $40 \mathrm{~L} \mathrm{~m}^{-2}$; no segundo, os de 60 e $80 \mathrm{~L} \mathrm{~m}^{-2}$ e, finalmente no último, os dados médios da disposição de 100 L m²; conclui-se, então, que após certo limite o aumento do volume aplicado de afluente levava a uma estratificação maior das concentrações de fósforo no efluente.

Quando se comparam esses dados médios com a legislação do Estado do Rio Grande do Sul (CONSEMA, 2006), que estabelecem o limite de $1 \mathrm{mg} \mathrm{L}^{-1}$ para emissão em um corpo hídrico constata-se que, em praticamente todas as situações o F025 apresentou resultados superiores a este valor. O filtro de maior profundidade, F100, foi capaz de manter o lixiviado abaixo desta normatização até a aplicação de $60 \mathrm{~L} \mathrm{~m}^{-2}$ na etapa 2, enquanto na etapa 3 este mesmo filtro já tinha um líquido inadequado desde a aplicação de $40 \mathrm{~L} \mathrm{~m}^{-2}$, chegando a atingir a concentração de $6,5 \mathrm{mg} \mathrm{L}^{-1}$, quando então se empregaram $100 \mathrm{~L} \mathrm{~m}^{-2}$.

Quanto aos percentuais de remoção, mais uma vez se constata a grande eficiência dos filtros de areia; na primeira etapa, os F050, F075 e F100 sempre apresentaram diminuição na concentração superior a 92,6\%, sendo que o F100 nunca teve resultados inferiores a 97,3\%. Esta alta remoção pode ser explicada, segundo Arias et al. (2001), Rodgers et al. (2005) e Sovik \& Klove (2005), pelo fato de ter havido uma ação biológica associada à provável presença de concentrações de ferro, alumínio ou calcário, que permite alta capacidade de retenção de fósforo.

\section{Escherichia coli}

Nota-se, nos filtros de areia e por meio da Tabela 7, que na aplicação de 20, 40 e $60 \mathrm{~L} \mathrm{~m}^{-2}$ durante as três etapas cada um apresentava densidades significativamente diferentes entre si; no emprego de 80 e $100 \mathrm{~L} \mathrm{~m}^{-2}$, nas etapas 2 e 3 o
F025 e o F050 tiveram tratamentos semelhantes, enquanto o F100 apresentava, em todas as situações, as menores densidades embora em alguns casos os valores encontrados estivessem acima de $4000 \mathrm{NMP} 100 \mathrm{~mL}^{-1}$, limite estabelecido pelo Estado de São Paulo para o lançamento em corpos hídricos, de Classe 3 (CETESB, 1976). Assim se conservou que, de forma geral, a afirmação de Stevik et al. (2004), Ausland et al. (2002) e Bancolé et al. (2003), para os quais quanto maior a profundidade do leito de areia, maior é o tempo de detenção do afluente no interior do reator, possibilitando um aumento da remoção dos microrganismos patogênicos devido principalmente à retenção e a degradação.

Tabela 7. Densidades médias de E Coli (NMP $\left.100 \mathrm{~m} \mathrm{~L}^{-1}\right)^{(1)(2)}$

\begin{tabular}{ccccrr}
\hline $\begin{array}{c}\text { Carga } \\
\text { (L m }\end{array}$ - $)$ & $\begin{array}{c}\text { Efluente } \\
\text { Anaeróbio }\end{array}$ & F025 & F050 & F075 & \multicolumn{1}{c}{ F100 } \\
\multicolumn{5}{c}{ Etapa 1 - 1 Aplicação por dia } \\
20 & $3,5 \times 106$ & $1,3 \times 104 \mathrm{aA}$ & $2 \mathrm{bA}$ & $3,2 \mathrm{cA}$ & $1,0 \mathrm{dA}$ \\
40 & $9,2 \times 106$ & $1,4 \times 106 \mathrm{aB}$ & $1,4 \times 104 \mathrm{bBC}$ & $460 \mathrm{cB}$ & $2,6 \mathrm{dA}$ \\
60 & $1,1 \times 107$ & $2,1 \times 106 \mathrm{aB}$ & $1,2 \times 105 \mathrm{bC}$ & $2,8 \times 104 \mathrm{cC}$ & $840 \mathrm{~dB}$ \\
80 & $7,5 \times 106$ & $4,5 \times 105 \mathrm{aB}$ & $2,8 \times 105 \mathrm{aD}$ & $7,4 \times 104 \mathrm{bD}$ & $1400 \mathrm{cC}$ \\
100 & $5,7 \times 106$ & $7,8 \times 104 \mathrm{aB}$ & $2,6 \times 104 \mathrm{bB}$ & $2,4 \times 104 \mathrm{cC}$ & $440 \mathrm{dBC}$ \\
\hline \multicolumn{5}{c}{ Etapa 2-2 Aplicações por dia } \\
20 & $1,2 \times 106$ & $860 \mathrm{aA}$ & $160 \mathrm{bA}$ & $49 \mathrm{cA}$ & $1,0 \mathrm{dA}$ \\
40 & $1,8 \times 107$ & $3,8 \times 105 \mathrm{aB}$ & $6,1 \times 104 \mathrm{bB}$ & $8700 \mathrm{cB}$ & $2200 \mathrm{~dB}$ \\
60 & $1,5 \times 107$ & $2,0 \times 106 \mathrm{aB}$ & $2,0 \times 105 \mathrm{bC}$ & $1,0 \times 105 \mathrm{cC}$ & $6500 \mathrm{dC}$ \\
80 & $1,4 \times 107$ & $3,7 \times 106 \mathrm{aB}$ & $1,7 \times 106 \mathrm{aD}$ & $5,7 \times 105 \mathrm{bD}$ & $1,4 \times 105 \mathrm{cD}$ \\
100 & $8,6 \times 106$ & $3,1 \times 106 \mathrm{aB}$ & $1,9 \times 106 \mathrm{aD}$ & $1,7 \times 106 \mathrm{bD}$ & $7,9 \times 105 \mathrm{cE}$ \\
\hline \multicolumn{5}{c}{ Etapa 3 - 3 Aplicações por dia } \\
20 & $8,1 \times 106$ & $9,7 \times 104 \mathrm{aA}$ & $1,5 \times 104 \mathrm{bA}$ & $470 \mathrm{cA}$ & $1,3 \mathrm{dA}$ \\
40 & $5,6 \times 106$ & $7,3 \times 105 \mathrm{aAB}$ & $2,6 \times 104 \mathrm{bA}$ & $1,2 \times 104 \mathrm{cB}$ & $150 \mathrm{~dB}$ \\
60 & $6,9 \times 106$ & $3,9 \times 106 \mathrm{aC}$ & $6,8 \times 105 \mathrm{bB}$ & $2,3 \times 105 \mathrm{cC}$ & $5,2 \times 104 \mathrm{dC}$ \\
80 & $4,8 \times 106$ & $3,3 \times 106 \mathrm{aBC}$ & $2,9 \times 106 \mathrm{aB}$ & $1,4 \times 106 \mathrm{bD}$ & $8,2 \times 105 \mathrm{bD}$ \\
100 & $3,1 \times 106$ & $3,6 \times 106 \mathrm{aBC}$ & $2,0 \times 106 \mathrm{aB}$ & $2,7 \times 106 \mathrm{aD}$ & $9,0 \times 105 \mathrm{bD}$ \\
\hline
\end{tabular}

${ }^{1}$ Médias seguidas da mesma letra minúscula na horizontal não diferem entre si (Tukey $5 \%$ ); ${ }^{2}$ Médias seguidas da mesma letra maiúscula na vertical não diferem entre si em uma mesma etapa (Tukey 5\%)

Avaliando-se cada filtro no decorrer do aumento das cargas aplicadas, nota-se que na etapa 1 os F075 e F100 tiveram no mínimo quatro níveis com densidades significativamente diferentes. O F025 possuía apenas dois níveis, em que os valores encontrados para os efluentes nas aplicações superiores a $40 \mathrm{~L} \mathrm{~m}^{-2}$ eram semelhantes estatisticamente, não demonstrando boa eficiência na remoção natural desses microrganismos; já para a aplicação de $100 \mathrm{~L} \mathrm{~m}^{-2}$ na terceira etapa, os F025, F050 e F075 tiveram líquidos lixiviados sem diferenças significativas em suas densidades.

Percebe-se que, em grande parte, os resultados estavam acima dos valores permitidos pela legislação paulista; ressalta-se, no entanto, que a remoção propiciada permitiria que para a adequação deste efluente a norma legal deverá ser feita com o gasto de menor quantidade de um agente desinfetante.

\section{CONCLUSÕES}

1. Quanto maior a profundidade do leito do filtro de areia maior também a capacidade de tratamento da matéria orgânica.

R. Bras. Eng. Agríc. Ambiental, v.14, n.2, p.227-234, 2010. 
2. As camadas de areia propiciaram, na maioria das situações, completa nitrificação, levando as altas concentrações de nitrato ao efluente.

3. As concentrações de fósforo foram baixas no efluente do filtro com 1,00 m de espessura de areia; no entanto, a profundidade de $0,25 \mathrm{~m}$ tinha concentrações superiores às estabelecidas na legislação do Estado do Rio Grande do Sul.

4. Em praticamente todas as situações avaliadas as densidades de E. Coli se mantinham acima da legislação pertinente.

\section{AGRADECIMENTOS}

À FAPESP, CNPq, FINEP, Caixa Econômica Federal e PROSAB, pelo apoio e financiamento na construção do projeto e em sua manutenção.

\section{LITERATURA CITADA}

Alagoas. Decreto n.6.200 de 1 de março de 1985. Diário Oficial do Estado de Alagoas. Maceió, 1985. 7p.

APHA/WEF - American Public Health Association/AWWA American Water Works Association/WEF - Water Environment Federation (WEF). Standard methods for the examination of water and wastewater. 19.ed. Nova Iorque: APHA/AWWA/ WEF, 1998. 1268p.

Arias, C. A.; Del Bubba, M.; Brix, H. Phosphorus removal by sands for use as media in subsurface flow constructed reed beds. Water Research, v.35, n.5, p.1159-1168, 2001.

Ausland, G., Stevik, T. K., Hanssen, J. F., Kohler, J. C., Jenssen, P. D. Intermittent filtration of wastewater: Removal of fecal coliforms and fecal streptococci. Water Research, n.36, p.3507-3516, 2002.

Bancolé, A.; Brissaud, F.; Gnagne, T. Oxidation processes and clogging in intermittent unsaturated infiltration. Water Science and Technology, v.48, n.11, p.139-146, 2003.

Brasil. Ministério do Meio Ambiente. Resolução CONAMA n.357, de 17 de março de 2005. Brasília: Diário Oficial da União, 2005. 23p.

Camargo, S. A. R.; Nour, E. A. A. Bamboo as an anaerobic medium: effect of filter column height. Water Science and Technology, v.44, n.4, p.63-70, 2001.

CETESB - Companhia de Tecnologia de Saneamento Ambiental. Decreto n.8.468 de 8 de novembro de 1976. Diário Oficial do Estado de São Paulo. São Paulo, 1976. 106p.

Check, G. G.; Waller, D. H.; Pask, D. A. The lateral-flow sand-filter system for septic-tank effluent treatment. Water Environmental Research, n.66, p.919-928, 1994.

CONAMA - Conselho Nacional do Meio Ambiente. Ministério do Meio Ambiente. Resolução CONAMA n.357 de 17 de março de 2005. Brasília: Diário Oficial da União, 2005. 23p.

CONSEMA - Conselho Estadual do Meio Ambiente. Resolução n.128 de 24 de novembro de 2006. Porto Alegre: Diário Oficial do Estado do Rio Grande do Sul, 2006. 9p.
Faria, L. C.; Pasquini, C. Flow-injection determination of inorganic forms of nitrogen by gas diffusion and conductimetry. Analytical Chemical Acta, v.245, p.183-190, 1991.

Foster, S. Poluição das águas subterrâneas: um documento executivo da situação da América Latina e Caribe com relação ao abastecimento de água potável. São Paulo: Instituto Geológico, 1993. 70p.

Hamoda, M. F.; Al-Ghusain, I.; Al-Mutairi, N. Z. Sand filtration of wastewater for tertiary treatment and water reuse. Desalination, v.164, p.203-211, 2004.

IBGE - Instituto Brasileiro de Geografia e Estadística. Pesquisa Nacional de Saneamento Básico. http://www.ibge.gov.br. 11 Abr. 2007.

Kristiansen, R. Sand-filter trenches for purification of septic tank effluent: II. The fate of nitrogen. Journal of Environmental Quality, v.10, p.358-361, 1981.

Oliveira, F. C.; Mattiazzo, M. E.; Marciano, C. R.; Moraes, S. O. Percolação de nitrato em Latossolo Amarelo Distrófico afetada pela aplicação de composto de lixo urbano e adubação mineral. Revista Brasileira de Ciência do Solo, v.35, p.731-742, 2001.

Pell, M.; Nyberg, F. Infiltration of wastewater in a newly started pilot sand-filter system: I Reduction of organic matter and phosphorus. Journal of Environmental Quality, v.18, p.451-457, 1989.

Prochaska, C. A.; Zouboulis, A. I. Performance of intermittently operated sand filters: a comparable study, treating wastewater of different origins. Water, Air and Soil Pollution, n.147, p.367-388, 2003.

Rodgers, M.; Healy, M. G.; Mulqueen, J. Organic carbon removal and nitrification of high strength wastewaters using stratified sand filters. Water Research, v.39, n.14, p.3279-3286, 2005.

Silva, G. H. R.; Nour, E. A. A. Reator compartimentado anaeróbio/aeróbio: Sistema de baixo custo para tratamento de esgotos de pequenas comunidades. Revista Brasileira de Engenharia Agrícola e Ambiental, v.9, n.2, p.268-275, 2005.

Sousa, J. T.; Ceballos, B. S. O.; Henrique, I. N.; Dantas, J. P.; Lima, M. S. Reúso de água residuária na produção de pimentão (Capsicum annuum L.). Revista Brasileira de Engenharia Agrícola e Ambiental, v.10, n.1, p.89-96, 2006.

Sovik, A. K.; Klove, B. Phosphorus retention processes in shell sand filter systems treating municipal wastewater. Ecological Engineering, v.25, n.2, p.168-182, 2005.

Stevik, T.; Ausland, A, K.; G.; Hanssen, J. Retention and removal of pathogenic bacteria in wastewater percolating through porous media: A review. Water Research, v.38, n.6, p.1355-1367, 2004.

Tonetti, A. L.; Coraucci Filho, B.; Stefanutti, R.; Figueiredo, R. F.; São Pedro, C. C. O. Remoção de matéria orgânica, coliformes totais e nitrificação no tratamento de esgotos domésticos por filtros de areia. Revista de Engenharia Sanitária e Ambiental, v.10, n.3, p.209-218, 2005.

Zonta, E. P.; Machado, A. A.; Silveira Junior, P. Sistemas de análises estatística para microcomputadores (SANEST). Pelotas: UFPel, 1984. 151p. 